\title{
¿Se debería enseñar a los estudiantes de los pregrados de economía el modelo de equilibrio general dinámico estocástico (DSGE)?
}

Álvaro Moreno Rivas*

Tal vez Sí. Tal vez No. Tal vez No importa.

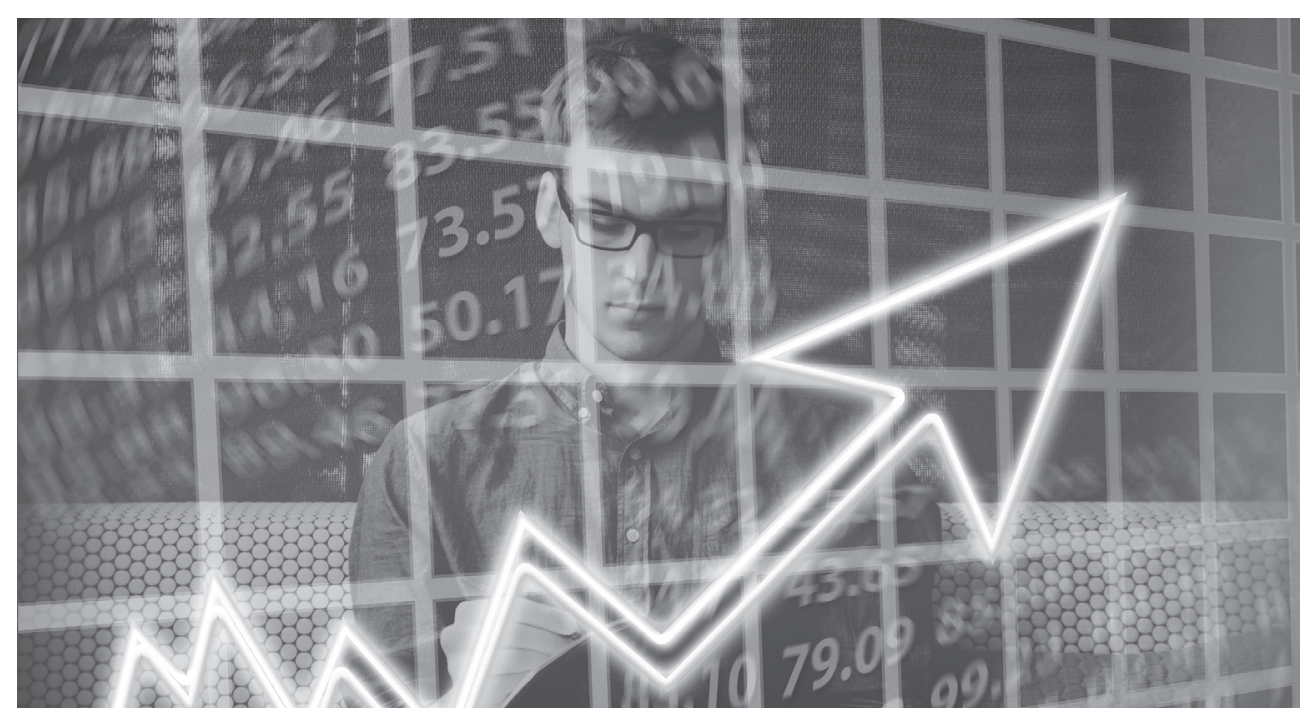

\section{Resumen}

En este artículo se presentan las consecuencias de introducir en los pregrados de economía la enseñanza avanzada de los modelos DSGE (equilibrio general dinámico estocástico). Esta monocultura conduce a la profundización de la insularidad de la disciplina, a la desaparición de la pluralidad paradigmática en los departamentos y al silenciamiento de las voces críticas. Este proceso obedece a la Ley de Gresham de las ideas: los malos modelos desplazan las buenas ideas.

Palabras clave: modelos DSGE, macroeconomía, política económica, macroeconomía moderna.

JEL: A22; E10; E60; E30

Citar este artículo como: Moreno Rivas, Á. (2018). ¿Se debería enseñar a los estudiantes de los pregrados de economía el modelo de equilibrio general dinámico estocástico (DSGE)? Revista Papeles, 10(19), 18-25.

Fecha recibido: noviembre 2017

fecha aceptación: abril 2018

* Profesor Asociado de la Universidad Nacional de Colombia y Profesor-Investigador de la Universidad Externado de Colombia. Correo electrónico: amoreno65@yahoo.es 


\section{Introducción}

Al final del tercer acto de la obra de teatro Jacques y su Amo de Milan Kundera (2005, p. 150), ambos personajes se enfrascan en una enigmática discusión. Jacques le pide a su Amo que lo guíe hacia delante, pero este le replica, “dónde es adelante?”. Sin dudarlo, Jacques le responde: "Adelante es hacia todas partes". Como toda gran literatura, la enseñanza es un viaje compartido, donde el maestro y sus discípulos emprenden una travesía intelectual. Infortunadamente, los profesores de la mainstream en economía ignoran la "secular astucia de la humanidad": "Adelante es hacia todas partes".

Los profesores jóvenes de macroeconomía sufren de una alta preferencia intertemporal. Consideran que la brecha existente entre los cursos que se dictan en el pregrado y los que se toman en los programas de maestría y doctorado debería cerrarse rápidamente. Se quejan por la falta de rigor y fundamentación microeconómica de la mayoría de los libros de texto intermedios y de la persistencia del modelo IS-LM ${ }^{1}$. Como respuesta a dicha situación, algunos docentes de la disciplina insisten en la necesidad de abrir un curso donde se inicie a los futuros economistas en el núcleo de la macroeconomía moderna, los modelos DSGE (Solis-García, 2018; Torres, 2014; Costa Junior, 2016).

Defender dicho programa y justificar su enseñanza en los pregrados como única alternativa para alcanzar la frontera de la investigación, claramente, es un ejercicio de propaganda y sectarismo intelectual. Sin duda, existen otros modelos rigurosamente fundamentados cuya riqueza analítica y conceptual compiten en los mismos escenarios con los modelos DSGE. Nos referimos a los modelos basados en agentes heterogéneos post-Keynesianos, los modelos Stock-Flujo consistentes de Godley y Lavoie

1 En general, se exceptúan dos libros: Barro (1986) y Williamson (2008).
(Veneziani y Zamparelli, 2018) y los modelos macrodinámicos derivados de la gran síntesis de Marx-Keynes-Schumpeter (Flaschel, 2009).

$\mathrm{Al}$ parecer, existe una demanda insatisfecha que se tiene que cubrir. Según los apologistas de los modelos DSGE, un curso introductorio de técnicas y matemáticas de optimización y computación se justifica desde la perspectiva del estudiante representativo por las tres siguientes razones:

Primero, para ayudar a los estudiantes para que trabajen tan cerca como sea posible a la frontera de la investigación en macroeconomía -los estudiantes deberían acceder a las revistas top y a los artículos del campo- los cuales están lejos de ser triviales. Segundo, para incrementar su capital humano y mejorar sus prospectos después de la graduación. $Y$ finalmente, porque en lo profundo de nuestros corazones esperamos que esos estudiantes, cuando sean introducidos a la frontera de la macroeconomía moderna, decidan que una vida de investigación es una vida que vale la pena vivir y se muevan al excitante proceso de obtener un PHD en economía. Nosotros podemos solamente soñar" (Solis-García, 2018, p. 227).

Los profesores de macroeconomía conmueven por su candor. Para ellos, las escuelas de economía están compuestas de un continuo de jóvenes-agentes homogéneos, cuyas preferencias y restricciones definen sus decisiones bajo condiciones competitivas e información imperfecta. Existe una fricción que impide que los muchachos y las muchachas puedan acceder al conocimiento científico de la frontera en macroeconomía.

El director del departamento actúa como un "dictador benevolente" que busca maximizar la función de utilidad de los estudiantes. En este sentido, diseña estrategias para corregir las 
fricciones que impiden la asignación eficiente de los talentos individuales. El mercado de las ideas debe resolver la falta de dicho activo.

La apertura de un curso de modelos DSGE permite que los estudiantes redefinan sus decisiones de inversión en capital humano, tomando en cuenta esta nueva "opción" que no solo mejorará sus destrezas técnicas y conceptuales sino que, además, garantiza que el rendimiento esperado compense el sacrificio de sueño, ocio y vagancia; recursos trasladados a largas horas de estudio de metodologías formalmente difíciles. Por su parte, las escuelas de economía garantizarán que sus programas avanzados de postgrado se llenen de los mejores candidatos. La oferta crea su propia demanda. Y el sueño está consumado.

Sin embargo, el mundo de "nunca jamás" que describen los modelos DSGE no es necesariamente la mejor "opción" para entender el funcionamiento de las economías capitalistas realmente existentes. Insistir en que lo único que se tiene que enseñar es el paradigma neoclásico o el llamado nuevo consenso macroeconómico con toda su filigrana técnica, es renunciar abiertamente al pluralismo en los departamentos de economía y ahondar la insularidad de la economía y de la macroeconomía en particular, levantando altos muros de contención.

Importantes autores de la corriente principal reconocen que los modelos DSGE son erróneos y que lo que se requiere es abrir las fronteras a modelos y a otras tradiciones, que si bien sobreviven en los bajos mundos de la "resistencia académica", también cuentan con rigor matemático y computacional (Blanchard, 2017; Haldane y Turrel, 2017). El tiempo es tal vez el único recurso verdaderamente escaso para un estudiante de economía. No se puede olvidar la regla de oro de la teoría del portafolio: "No ponga todos los huevos en una sola canasta".

Este artículo pretende mostrar que lo que se busca con la introducción de un curso de "frontera" de modelos DSGE en la macroeconomía del pregrado es prácticamente eliminar el pluralismo teórico y metodológico de la enseñanza. Es curioso que los economistas no tomen en consideración los problemas de reducir los programas de enseñanza a una monocultura formal y analítica que aún no responde adecuadamente a las fallas presentes en sus fundamentos y principios heurísticos (Rogers, 2008).

Vale la pena reseñar el malestar intelectual que se vive en un área tan reputada como la teoría de la gravedad cuántica. Después de la consolidación de la teoría de la relatividad general (TRG) y de la mecánica cuántica (MC), los físicos teóricos se vieron en la necesidad de buscar una teoría que las integrara a ambas de una manera consistente (Rovelli, 1999).

La TGR transformó radicalmente nuestra concepción del espacio y del tiempo, pero mantuvo la visión newtoniana de la relación entre el observador y objeto observado. Por el contrario, la MC dejó sin cuestionar las ideas de espacio-tiempo de la física clásica, pero cambió las relaciones entre el observador y el objeto de la observación (Smolin, 2001).

A lo largo del siglo XX surgieron tres programas de investigación en el campo de la física: la teoría de cuerdas, la teoría cuántica de bucles y la teoría de Twistor. A pesar de que las tres teorías han reportado importantes avances en el proyecto, aún no se cuenta

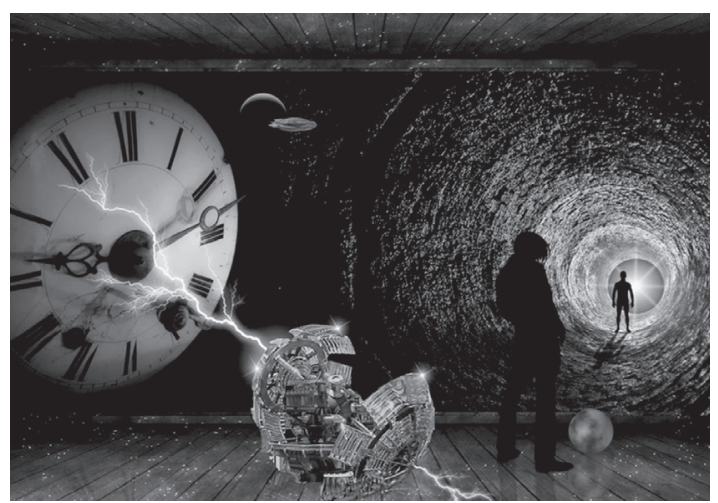


con la teoría final. Sin embargo, la teoría de cuerdas, por diversas razones, se consolidó como la mainstream, desplazando a las otras alternativas igualmente rigurosas y respetables, provocando un grave estancamiento en el desarrollo de las ideas y una larga ralentización en la tasa de nuevos descubrimientos (Smolin, 2006). Algo similar ocurre en la disciplina económica con los modelos DSGE, que se constituyen como la nueva síntesis neoclásica que integra los modelos RBC y la macroeconomía neokeynesiana.
Este artículo consta de cuatro partes: la primera es esta introducción. La segunda presenta la propuesta y el enfoque de un curso estándar de la frontera en macroeconomía de modelos DSGE. La tercera presenta un modelo donde se simulan las consecuencias nefastas de enseñar los modelos de equilibrio general dinámico estocástico (DSGE) - como si fuera la mejor teoría económica - para la continuidad del pluralismo y la sobrevivencia de las voces críticas en los departamentos de economía. Finalmente se derivan unas breves conclusiones.

\section{Cocinando modelos gourmet DSGE en un curso de pregrado}

Tal vez la mejor manera de entender la importancia que le otorgan los profesores de macroeconomía a la enseñanza de los modelos $D S G E$ sea comparar un curso de estos con el aprendizaje de la culinaria gourmet. Aprender a cocinar es una tarea que requiere esfuerzo y estudio denso. Las evaluaciones son individuales y reflejan la capacidad para seguir recetas y combinar ingredientes que sorprendan a los comensales. Para destacarse se requiere que el estudiante sea capaz de hacer pequeñas variaciones a la receta "núcleo" con el fin de obtener un plato diferenciado. Se pueden hacer fusiones e innovaciones, pero conservando la estructura básica de la receta y los pasos de la preparación.

Aprender cómo se construyen modelos DSGE es como aprender a preparar un plato de "alta cocina". Usted debe dominar la técnica, conocer las recetas y combinar los ingredientes para obtener un plato nuevo que responda al paladar de la comunidad de macroeconomistas de "frontera".

Los ingredientes pueden variar, pero no se salen de los siguientes: un continuo de hogares consumidores cuyas preferencias los convierten en clones. Un continuo de firmas que enfrentan condiciones homogéneas de tecnología y que pueden actuar bajo condiciones de competencia perfecta o competencia monopolista. Un gobierno o un banco central. También se pueden incluir bancos y un sector externo. Las salsas son variadas y las llaman "fricciones"; es decir, pequeñas cuñas que impiden que los mercados funcionen eficientemente.

Dependiendo del tipo de modelo a construir, se asume la flexibilidad de precios o se introducen rigideces nominales o reales, todo al gusto del consumidor. Todos los agentes resuelven problemas de optimización y se asume que lo hacen bajo la hipótesis de expectativas racionales. Los modelos cumplen con la exigencia de estar microfundamentados, de ser de equilibrio general, de ser dinámicos y estar sometidos a perturbaciones exógenas estocásticas.

Todo este programa usted lo puede aprender en un curso de apenas 14 semanas, con tres horas de lectura y una hora y media de laboratorio a la semana. Necesita únicamente conocer las técnicas de optimización multivariada bajo restricciones, haber aprobado los cursos de macroeconomía intermedia (Williamson, 2008). Aunque sería bueno tener conocimientos de computación (no es necesario).

El curso que propone Mario Solis-García (2018) es el siguiente:

Semanas 1-2: equilibrio competitivo

Semanas 2-3: solución de ecuaciones en MATLAB

Semanas 3-5: programación dinámica

Semanas 5-7: técnicas de log-linealización 
Semanas 8-10: aplicaciones (oferta laboral, política fiscal, otros tópicos)

Semanas 11-13: econometría bayesiana, calibración y dynare

Semanas 13-14: aplicaciones (parametrización modelo de Hansen y modelos neokeynesianos)

Sin duda alguna, el curso de cocina gourmet de modelos DSGE enseña a cocinar modelos DSGE. Los estudiantes quedarán convencidos de que lo único que vale la pena cocinar son modelos DSGE. Una vez ingresen a un PhD considerarán que el mejor camino para obtener una tesis laureada es cocinar un modelo DSGE y cuando obtengan una plaza de profesor en una universidad terminarán enseñando a cocinar modelos DSGE.

En el departamento de economía nadie habla de otra cosa que no sea de modelos DSGE, y quien desafíe la hegemonía de los modelos DSGE será señalado como un agente que al parecer no hizo la inversión correcta. El "nosotros" propio de los cuarteles y del seminario religioso expulsará al intruso. En ese momento habrá desparecido la pluralidad de la enseñanza y la crítica de la discusión académica. Resultado: estancamiento secular del departamento.

El éxito de este tipo de cursos reside en el desarrollo de habilidades y destrezas técnicas. La inversión de los estudiantes debe definirse como un costo hundido; es decir, convierte la decisión en irreversible. Como dicen los economistas de la organización industrial: "una barrera a la entrada se convierte en una barrera a la salida”. Después de que un joven invierte gran parte de sus recursos y su tiempo en aprender DSGE es prácticamente imposible que se ocupe de otra cosa y deseche cínicamente los serios cuestionamientos y problemas de la teoría básica que los sustentan.

Nadie arroja por la borda lo que le ha costado tanto aprender y no dudará en descartar cualquier crítica o cuestionamiento a su trabajo, preso de la disonancia cognitiva. Como lo manifiesta Mark Setterfield (2018), un eminente profesor de macroeconomía de la New School for Social Research ${ }^{2}$ :

Habiendo reducido a los tomadores de decisiones a un solo agente representativo, la macroeconomía de la corriente principal moderna también falla en prestarle atención a lo aprendido en la teoría del equilibrio general de hace 40 años. Los celebrados teoremas de Sonnenschein-Debreu-Mantel (SDM) muestran que la economía en su conjunto es improbable que refleje las propiedades de buen comportamiento que se asumen para los individuos. En particular, si existe alguna heterogeneidad

2 Having reduced decision-makers to a single representative agent, modern mainstream macroeco- nomics also fails to heed lessons learned in general equilibrium theory over 40 years ago. The celebrated Sonnenschein-Debreu-Mantel (SDM) results show that the entire economy is unlikely to reflect the "well-behaved" properties assumed of individuals. In particular, if there is any heterogeneity among agents, then all bets are off as regards the uniqueness and stability of economywide equilibrium. The representative agent is thus revealed as evading rather than solving critical problems of aggregation (the relationship between parts and wholes), unless the economy is made up of a single Robinson- Crusoe-like figure or, alternatively, a plurality of clones (it isn't). None of this would have been news to Keynes, Mitchell, or any of the architects of the structural macroeconomics (then or now) that DSGE advocates (if not Solis-Garcia himself) seek to purge from the undergraduate curriculum. There always was (and still is) an awareness among structuralists that the economy is made up of parts (households, firms, social classes, markets), and that generalizations about aggregate phenomenon must be accompanied by plausible claims about the behavior of these parts. The argument is that no simple set of relation-ships links parts and wholes, there being (in particular) no obvious possibility of reducing explanation of the macro whole to its constituent micro parts. Keynes's famous paradox of thrift was an early indication of this, as were the "perverse" macro results that followed from individual firms' behavior in Harrod's dynamics. All of this is now widely recognized as being part of the universe of emergent properties that makes the pursuit of reductionism futile. 
entre los agentes, entonces todas las apuestas están perdidas en cuanto a la unicidad y estabilidad del equilibrio de la economía. El agente representativo se revela más como una evasión que como una solución al problema crítico de agregación (la relación entre las partes y el todo), a menos que la economía cuente con un solo agente Robinson-Crusoe-como figura o, alternativamente, una pluralidad de clones. Nada de esto había sido nuevo para Keynes, Mitchell, o cualquier otro de los arquitectos de la macroeconomía estructuralista (entonces o ahora) que han defendido los DSGE (si no Solis-García mismo) y han buscado purgar de los currículos del pregrado. Siempre existió un cuidado entre los estructuralistas que la economía se compone de partes (hogares, firmas, clase sociales, mercados) y que las generalizaciones sobre los fenómenos agregados deben ser acompañados por plausibles demandas acerca del comportamiento de esas partes. El argumento no es establecer relaciones simples entre el todo y las partes, no es tan obvio reducir la explicación de la totalidad macro a sus partes micro que la constituyen. La famosa paradoja del ahorro de Keynes fue una de las primeras indicaciones sobre este tipo de problemas, como lo fue el resultado perverso macro que se sigue de las conductas individuales de las firmas en la dinámica de Harrod. Todo esto es ahora ampliamente reconocido por ser parte del universo de propiedades emergentes que hace al reduccionismo fútil (Setterfield, 2018, p. 239) ${ }^{3}$.

\section{Después de que un joven invierte gran parte de sus recursos y su tiempo en aprender DSGE es prácticamente imposible que se ocupe de otra cosa y deseche cínicamente los serios cuestionamientos y problemas de la teoría básica que los sustentan.}

\section{Enseñanza de modelos DSGE y pluralismo en economía}

El objetivo en este acápite es presentar un modelo evolucionista de lucha paradigmática entre dos escuelas en el seno de un departamento de economía (Palley, 1997). El sistema de ecuaciones responde una importante pregunta: ¿por qué algunas ideas se expanden y dominan el pensamiento económico, mientras que otras se desplazan al baúl de los cursos de historia de las doctrinas económicas? Existen dos tipos de profesores de economía. Los NC, que son los neoclásicos y los defensores de introducir la enseñanza de los modelos DSGE en los programas de pregrado. Los PK son los economistas heterodoxos, quienes defienden el pluralismo académico. Las ecuaciones son las siguientes:

$$
S_{t}^{N C}=a S_{t-1}^{N C}+(1-b) S_{t-1}^{P K}
$$

$$
S_{t}^{P K}=b S_{t-1}^{P K}+(1-a) S_{t-1}^{N C}
$$

$S_{t}^{N C}$ y $S_{t}^{P K}$ son las participaciones de los dos tipos de profesores-investigadores en el total de miembros del departamento. Por definición, $S_{t}^{N C}+S_{t}^{P K}=1$.

Los parámetros del sistema están acotados de la siguiente manera: $0 \leq a \leq 1$ y $0 \leq b \leq 1$. En el modelo, "a" y "b" representan las prácticas sociales y las reglas de juego en el departamento. Por ejemplo, el departamento promueve o no el pluralismo, existen controles y filtros sesgados en contra de algún grupo en las publicaciones y financiamiento de los proyectos de investigación o se privilegian ciertos cursos sobre otros.

La traducción es del autor de este artículo. 
Gráfico 1. Solución Pluralista del Modelo

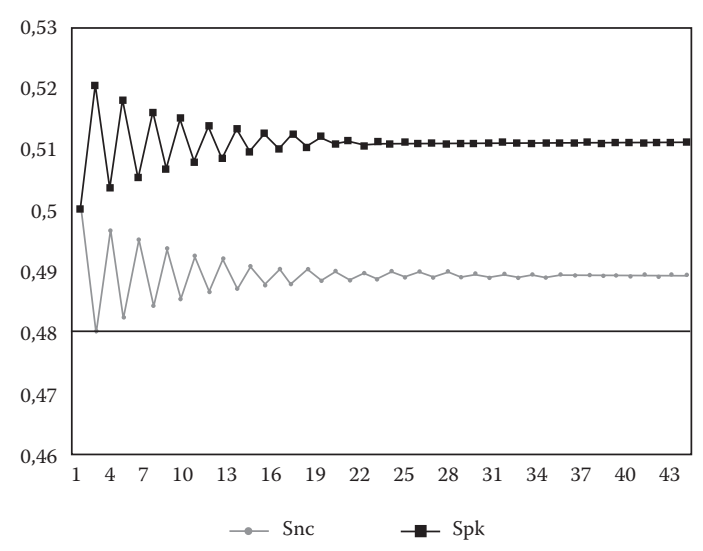

Fuente: cálculos propios

Un departamento que promueve la discusión y tolera la existencia de la diversidad paradigmática se caracteriza por tener valores de "a" $\mathrm{y}$ "b" menores que 1.

En el gráfico 1 se muestra la simulación de la interacción de los profesores-investigadores en un mundo académico abierto y tolerante. En el eje horizontal se registran los períodos o el tiempo, en el eje vertical se muestra la evolución de los porcentajes de cada tipo de profesor en la nómina total del departamento. Como se puede observar, en el largo plazo se conserva la diferencia teórica y la visión pluralista.

Cuando en el departamento se imponen las preferencias de las nuevas generaciones de profesores, educados en la "frontera" de los modelos DSGE, no solo cambian las correlaciones de fuerzas políticas sino que las reglas de juego y los criterios de evaluación y promoción se definen sobre nuevos indicadores y derroteros.

Quienes consideran que es necesario promover y generalizar las ideas de la corriente principal inician con una especie de acción paralela con el objetivo de reducir los cursos de historia, economía heterodoxa y áreas afines, reemplazándolos con "nuevos" cursos que se venden como papa caliente entre los miembros de la comunidad universitaria, ya que se presentan como el único camino al éxito profesional
Gráfico 2. Solución No Pluralista del Modelo

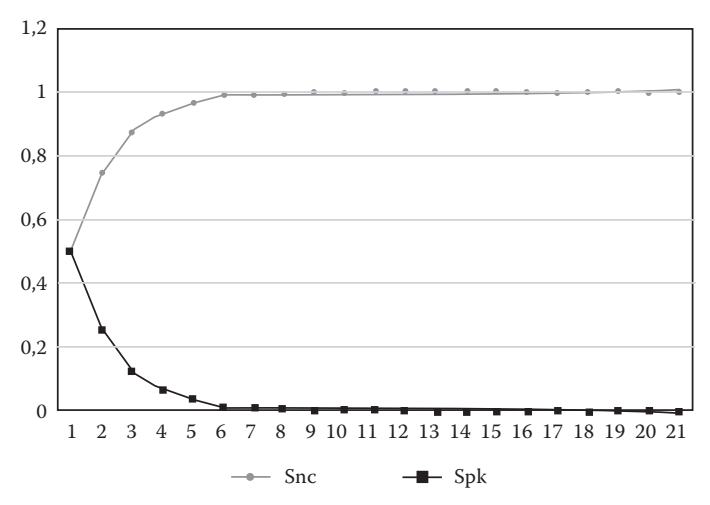

Fuente: cálculos propios

y científico. Se repite sin mayor criterio: los modelos DSGE son la verdad, el camino y la vida misma.

Bajo estas condiciones, el parámetro "a" es igual a 1. Como se muestra en el gráfico 2, la consecuencia de las nuevas reglas de juego académicas conducen a la desaparición de la pluralidad y la diversidad de enfoques en el departamento. Solamente sobreviven los profesores-investigadores neoclásicos.

\section{Conclusiones}

Las propuestas de introducir cursos avanzados para enseñar modelos DSGE en el pregrado de economía con el objeto de acercar a los estudiantes a la "frontera" de la investigación científica puede tener consecuencias agregadas para los departamentos que no se deducen de los beneficios microeconómicos de los estudiantes y de los profesores.

La prioridad de enseñar una monocultura terminará por destruir la discusión pluralista del sistema. En consecuencia, desaparecerán las voces críticas, y los disparates escritos en lenguaje matemático reemplazarán las buenas teorías. Es la ley de Gresham de las ideas: Los malos modelos desplazan a las buenas ideas.

Como dice Setterfield (2018): "Como todos saben, malas teorías conducen a malas 
políticas. Pero quizá lo peor de todo es que las malas teorías terminan en malas aptitudes políticas" (p. 239).

\section{Referencias}

Barro, R. (1986). Macroeconomía. México: Interamericana.

Blanchard, O. (2017). Do DSGE models have a future? In Refet S. Gürkaynak and Cédric Tille (Ed). DSGE Models in the conduct of policy. London: CEPR Press.

Costa, C. J. (2016). Understanding DSGE models. Theory and Applications. Malaga: Vernon Press.

Flaschel, P. (2009). The Macrodynamics of Capitalism. Elements for a Synthesis of Marx, Keynes and Schumpeter. Germany: Springer.

Kundera, M. (1981). Jacques y su Amo. Homenaje a Denis Diderot. Madrid: Tusquets Editores.

Haldane, A. \& Turrel, E. A. (2017). An interdisciplinary model for macroeconomics. Staff Working Papers, 6(96). London: Bank of England.

Palley, T. (1997). The Academic Jungle: social practice and the survival of economic ideas. Review of Radical Political Economy, 29(3), 22-33.
Rogers, C. (2008). Money as Friction: Conceptual Dissonance in Woodford's Interest and price. Research Paper, 2008(3).

Rovelli, C. (1999). Quantum spacetime: what do we know'. Marseille. France: Centre de Physique Theorique de Luminy.

Setterfield, M. (2018). Maybe you can, but perhaps you shouldn't!. Saving undergraduate macroeconomics from DSGE modeling. The Journal of Economic Education, 49(3), 237-241.

Smolin, L. (2001). Three roads to Quantum Gravity. New York: Basic Books.

Smolin, L. (2006). Las Dudas de la física en el siglo XXI. ¿Es la teoría de cuerdas un callejón sin salida? Barcelona: Crítica.

Solis-Garcia, M. (2018). Yes we can!. Teaching DSGE models to undergraduate students. The Journal of Economic Education, 49(3), 226-236.

Torres, J. L. (2014). Introduction to Dynamic Macroeconomic General Equilibrium Models. Málaga: Vernon Press.

Veneziani, R. \& Luca, Z. (2018). Analitycal Political Economy. New Delhi, India: Wiley Blackwell.

Williamson, S. (2008). Macroeconomics. New York: Pearson Addison Wesley. 